\title{
Correction to: Small Intestinal Length Associates with Serum Triglycerides Before and After LRYGB
}

\author{
Pirjo Käkelä ${ }^{1}$ Ville Männistö ${ }^{2} \cdot$ Maija Vaittinen ${ }^{3} \cdot$ Sari Venesmaa $^{1} \cdot$ Vesa Kärjä $^{4} \cdot$ Kirsi Virtanen $^{5} \cdot$ Hannu Paajanen $^{1}$. \\ Jussi Pihlajamäki ${ }^{3,5}$
}

Published online: 14 December 2021

๑) Springer Science+Business Media, LLC, part of Springer Nature 2021

\section{Correction to: Obesity Surgery (2018) 28:3969-3975 https://doi.org/10.1007/s11695-018-3447-x}

The ORCID iD https://orcid.org/0000-0002-6383-0779 is the ORCID iD of the author Pirjo Käkelä, not Jussi Pihlajamäki.

Publisher's Note Springer Nature remains neutral with regard to jurisdictional claims in published maps and institutional affiliations.

The original article can be found online at https://doi.org/10.1007/ s11695-018-3447-x.

Jussi Pihlajamäki

jussi.pihlajamaki@uef.fi

Pirjo Käkelä

pirjo.kakela@kuh.fi

Ville Männistö

ville.mannisto@kuh.fi

Maija Vaittinen

maija.vaittinen@uef.fi

Sari Venesmaa

sari.venesmaa@kuh.fi

Vesa Kärjä

vesa.karja@kuh.fi

Kirsi Virtanen

kirsi.virtanen@uef.fi
Hannu Paajanen

hannu.paajanen@kuh.fi

1 Department of Surgery, University of Eastern Finland and Kuopio, University Hospital, Kuopio, Finland

2 Department of Medicine, University of Eastern Finland and Kuopio, University Hospital, Kuopio, Finland

3 Institute of Public Health and Clinical Nutrition, University of Eastern Finland, 70210 Kuopio, Finland

4 Department of Pathology, University of Eastern Finland and Kuopio, University Hospital, Kuopio, Finland

5 Department of Clinical Nutrition and Obesity Centre, University of Eastern Finland and Kuopio, University Hospital, Kuopio, Finland 